\title{
Reflection and refraction process of spinwave in a ferromagnet/frustrated ferromagnet junction system
}

\author{
Yuta Sasaki and Hiroaki T. Ueda \\ Department of Physics, Tokyo Metropolitan University, Hachioji, Tokyo 192-0397, Japan
}

\begin{abstract}
Frustration introduces a nontrivial dispersion relation of spinwave even in a ferromagnetic phase in a spin system. We study the reflection and refraction process of spinwaves in the ferromagnet/frustrated ferromagnet junction system by using the Holstein-Primakoff spinwave expansion and taking the large- $S$ limit. We discuss the relation between the incident angle and the refraction angle of spinwave, namely, the Snell's law of spinwave. As concrete examples of frustrated ferromagnets, we study the fully polarized ferromagnet phases in $J_{1}-J_{2}$ chains and the $J_{1}-J_{2}$ models on the square lattice. The interesting refraction processes, such as the splitting of the incident spinwave and the negative refraction, are discussed. We also study the transmittance and reflectance in these concrete models.
\end{abstract}

KEYWORDS: spinwave, magnonics, junction system, frustration, negative refraction

\section{Introduction}

Spinwave is a collective excitation of spins in magnets. Since spinwave can carry information on the nano/microscale, recently, potential application of spinwave to new devices has attracted much attention to researchers. ${ }^{1)}$ In many circumstances, spinwave behaves like a sound or light wave; reflection and refraction process of spinwave can be considered.

Understanding of the reflection and refraction process is important to control spinwave. In the junction system of usual ferromagnets in which spinwave has a 'trivial' ${ }^{2)}$ dispersion relation, the reflection and refraction process is theoretically understood by using geometrical-optics approximation ${ }^{3-6)}$ or Landau-Lifshitz-Gilbert equation. ${ }^{7)}$ One of the important results of these studies is that the direction of the propagating spinwave depends on the strength of applied magnetic field. The experimental control of propagation of spinwave is indeed realized by tuning the internal demagnetizing fields in a permalloy waveguid. ${ }^{8)}$ If the dispersion relation of spinwave is nontrivial, an exotic refraction process is expected. In the case of light, for example, the negative refraction occurs due to the nontrivial dispersion relation. ${ }^{9,10)}$ Recently, it is theoretically proposed that the anisotropic nature of the dispersion relation of dipole-exchange spinwaves can result in the negative refraction. ${ }^{7)}$

Recent experimental advance has been exhibiting a wide variety of frustrated magnets. Magnetic frustration provides us with an exotic magnetic behavior. ${ }^{11)}$ Even in a ferromagnet, in which all spins align, the frustration can induce an unusual dispersion relation of spinwaves. Hence, it will be natural to expect interesting refraction processes of spinwaves in frustrated ferromagnets. In this paper, we study the reflection and refraction process of spinwave in ferromagnet/frustrated ferromagnet junction system.

As a concrete example of frustrated ferromagnets, we consider the spin systems which are already realized experimentally. For example, a frustrated ferromagnet can be prepared by applying high magnetic field on every frustrated magnet. One-dimensional (1D) $J_{1}-J_{2}$ Heisenberg chain with a nearest-neighbor exchange coupling $J_{1}$ and next-nearestneighbor coupling $J_{2}$ is one of the famous frustrated magnets. ${ }^{12-16)}$ In the fully polarized phase under high magnetic field, the dispersion of spinwave has two minima at ${ }^{12,17-19)}$ the wavevector $Q$ s of $\cos Q=-J_{1} / 4 J_{2}$ for $\left|J_{1}\right| / J_{2}<4$ and $J_{2}>0$. There are many compounds consisting of the $J_{1}-J_{2}$ chains (see Table. I in Ref. 13). The saturated ferromagnetic phases under high magnetic field are experimentally realized in ${ }^{13)} \mathrm{Rb}_{2} \mathrm{Cu}_{2} \mathrm{Mo}_{3} \mathrm{O}_{12},{ }^{20-22)}\left(\mathrm{N}_{2} \mathrm{H}_{5}\right) \mathrm{CuCl}_{3}$ and ${ }^{23-25)} \mathrm{LiCuVO}_{4}$.

As another example of the frustrated magnets, the various compounds of the square-lattice $J_{1}-J_{2}$ model, e.g., $\mathrm{BaCdVO}\left(\mathrm{PO}_{4}\right)_{2}$, are reported. ${ }^{26,27)}$ Without external field, the collinear antiferromagnet phase appears in $\mathrm{BaCdVO}\left(\mathrm{PO}_{4}\right)_{2}$; the saturation field is about $4 \sim 6 \mathrm{~T}$. In the saturated ferromagnetic phase of this compound, theoretically, the dispersion relation is expected to be nontrivial; it has two minima at $\mathbf{Q}=(0, \pi)$ and $\mathbf{Q}=(\pi, 0){ }^{26-28)}$

The organization of the present paper is as follows. In Sec. 2, we briefly review the dispersion relations of spinwaves in (frustrated) ferromagnets by using the Holstein-Primakoff spiwave expansion. In Sec. 3, we generally discuss refraction angles of transmission spinwaves in the ferromagnets junction systems satisfying the given conditions. Then, we apply this discussion to the several junction systems including the frustrated $J_{1}-J_{2}$ chains or the $J_{1}-J_{2}$ model on the square lattice. We shall explicitly see the splitting of the incident spinwave and the negative refraction in these models. In Sec. 4, we study the reflection- and the transmission rates (reflectance and transmittance) in part of the junction systems considered in Sec. 3 by solving the simple Schröedinger equations within the large $S$ limit. Our approach naturally treats the lattice structure of ferromagnets without the coarse graining.

\section{Dispersion Relation of Frustrated Ferromagnets}

As a brief review, let us discuss the dispersion relation of spinwaves in the fully-polarized ferromagnetic phase of (frustrated) spin systems. For simplicity, we consider the lattice systems with one magnetic ion per unit cell, and assume the rotational symmetry around the $z$ direction in spin space. This assumption leads to the conservation law of the total angular momentum along the $z$ direction, namely, $\sum_{\mathbf{i}}\left\langle S_{\mathbf{i}}^{z}\right\rangle$. We study the Hamiltonian with the generic exchange interactions $J_{\mathbf{i j}}=J_{\mathbf{i}-\mathbf{j}}=J_{\mathbf{j}-\mathbf{i}}=J_{\mathbf{l}}$, the on-site anisotropic interaction term 
$K$, and the external magnetic field $H$ :

$$
H=\sum_{\langle\mathbf{i}, \mathbf{j}\rangle} J_{\mathbf{i j}} \mathbf{S}_{\mathbf{i}} \cdot \mathbf{S}_{\mathbf{j}}+\sum_{i}\left(-K\left(S_{\mathbf{i}}^{z}\right)^{2}+\mathrm{H} S_{\mathbf{i}}^{z}\right),
$$

where we use the coordinate $(x, y, z)$ in spin space, and $\mathbf{i}=$ $\left(a_{i}, b_{i}, c_{i}\right)$ in lattice space. Let us rewrite the spin operator by using the Holstein-Primakoff transformation on the fullypolarized ferromagnetic phase:

$$
\begin{aligned}
& S_{\mathbf{i}}^{z}=-S+\alpha_{\mathbf{i}}^{\dagger} \alpha_{\mathbf{i}}, \\
& S_{\mathbf{i}}^{+}=\sqrt{2 S} \alpha_{\mathbf{i}}^{\dagger} \sqrt{1-\frac{\alpha_{\mathbf{i}}^{\dagger} \alpha_{\mathbf{i}}}{2 S}} \approx \sqrt{2 S} \alpha_{\mathbf{i}}^{\dagger}, \\
& S_{\mathbf{i}}^{-}=\sqrt{2 S} \sqrt{1-\frac{\alpha_{\mathbf{i}}^{\dagger} \alpha_{\mathbf{i}}}{2 S}} \alpha_{\mathbf{i}} \approx \sqrt{2 S} \alpha_{\mathbf{i}} .
\end{aligned}
$$

By using the $1 / S$ expansion, we approximately obtain the free bosonic Hamiltonian in the leading order in $1 / S$ :

$$
H=\sum_{\mathbf{k}}(\omega(\mathbf{k})-\mu) \alpha_{\mathbf{k}}^{\dagger} \alpha_{\mathbf{k}},
$$

where

$$
\begin{aligned}
\epsilon(\mathbf{k}) & =\frac{1}{2} \sum_{\mathbf{l}} J_{\mathbf{l}} \cos \mathbf{k} \cdot \mathbf{l}, \omega(\mathbf{k})=2 S\left(\epsilon(\mathbf{k})-\epsilon_{\min }\right), \\
\mu & =2 S\left(\epsilon(0)-\epsilon_{\min }\right)-(\mathrm{H}+2 S K),
\end{aligned}
$$

and $\epsilon_{\min }$ is the minimum of $\epsilon(\mathbf{k})$. The conservation law of the angular momentum along the $z$ direction assures the conservation of the total magnon number. For any exchange interactions, a sufficiently large $\mathrm{H}$ or $K$ can induce the gap $(\mu \leq 0)$ in the magnon dispersion. If $\mu \leq 0$, the ferromagnetic phase is stable. Throughout this paper, we focus on this free bosonic Hamiltonian by assuming the large $S$ limit.

We have seen that a dispersion relation takes various forms due to frustration. This dispersion relation can lead to the nontrivial group velocity not parallel to the phase velocity, where the group velocity is given by

$$
\mathbf{v}_{g}(\mathbf{k})=\nabla_{\mathbf{k}} \omega(\mathbf{k}) .
$$

The group velocity has an important physical meaning: it carry the angular momentum as reviewed in Appendix. Not the phase- but the group velocity determines the traveling direction of spinwave. Next, let us study the various dispersion relations in the concrete models.

\section{1 simple case}

First, we consider the simple Heisenberg model on the cubic lattice with the nearest neighbor ferromagnetic coupling $J<0$ as shown in Fig. 1. $\epsilon(\mathbf{k})$ is given by

$$
\begin{aligned}
& \epsilon_{s}(\mathbf{k})=J\left(\cos k^{a}+\cos k^{b}+\cos k^{c}\right), \\
& \epsilon_{s \min }=\epsilon_{s}\left(\mathbf{k}_{0}=(0,0,0)\right)=3 J .
\end{aligned}
$$

This leads to the dispersion relation $\omega_{s}(\mathbf{k})$ shown in Fig. 2. In the long wavelength limit $|\mathbf{k}| \rightarrow 0$, this dispersion relation becomes isotropic and is given by

$$
\omega_{s}(\mathbf{k}) \approx \frac{\mathbf{k}^{2}}{2 m_{s}}, m_{s}=-\frac{1}{J} .
$$

In the following discussion, we use this model as the concrete example of 'usual' ferromagnets.

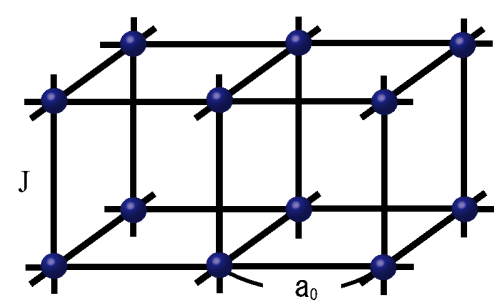

Fig. 1. (Color online) The cubic lattice with the nearest neighbor Heisenberg exchange coupling $J$. The dots represent spins. The lattice constant $a_{0}=1$ is assumed.

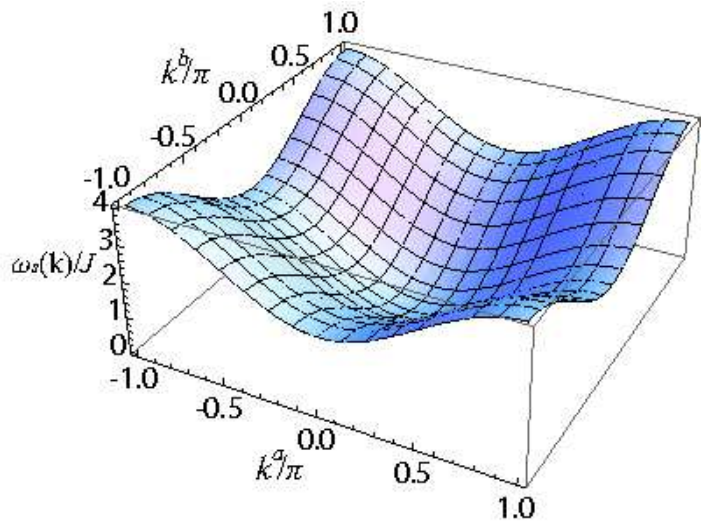

Fig. 2. (Color online) The dispersion relation $\omega_{s}(\mathbf{k})$ of the trivial ferromagnet on the cubic lattice for $J<0 . k^{c}=0$ is assumed.

\section{$2.2 J_{1}-J_{2}$ chain}

Next, let us discuss the dispersion relation of the 1D $J_{1}-J_{2}$ chains with the ferromagnetic interchain coupling $J_{3}<0$ on the cubic lattice ${ }^{17-19,29)}$ as illustrated in Fig. 3:

$$
\begin{aligned}
\epsilon_{1}(\mathbf{k}) & =J_{1} \cos k^{c}+J_{2} \cos 2 k^{c}+J_{3}\left(\cos k^{a}+\cos k^{b}\right) \\
& =2 J_{2}\left(\cos k^{c}+\frac{J_{1}}{4 J_{2}}\right)^{2}-\frac{J_{1}^{2}}{8 J_{2}}-J_{2}+J_{3}\left(\cos k^{a}+\cos k^{b}\right),
\end{aligned}
$$

where the $J_{1}-J_{2}$ chains are assumed to lie parallel to the $c$ axis. For $\left|J_{1}\right| / J_{2} \leq 4, J_{2}>0$ and $J_{3}<0$, the dispersion relation has two minima at $\mathbf{Q}=\left(0,0, \pm Q^{c}\right)$ where

$$
\cos Q^{c}=-\frac{J_{1}}{4 J_{2}}, \quad \epsilon_{\min }=-\frac{J_{1}^{2}}{8 J_{2}}-J_{2}+2 J_{3} .
$$

The dispersion relation $\omega_{1}(\mathbf{k})$ is graphically shown in Figs. 4,5.

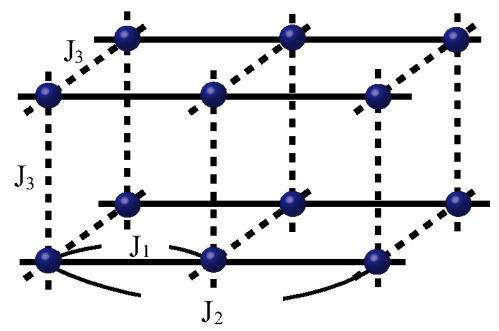

Fig. 3. (Color online) $J_{1}-J_{2}$ chains with the interchain coupling $J_{3}$ on the cubic lattice. The dots represent spins. 


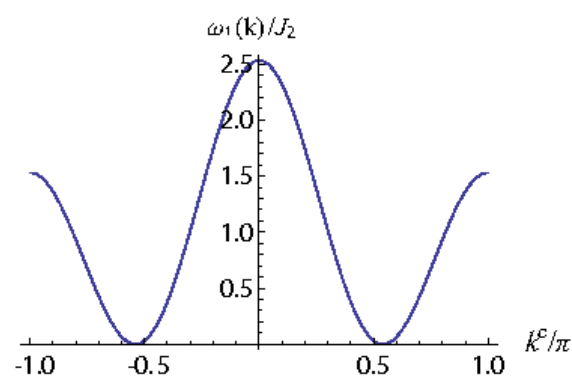

Fig. 4. (Color online) The dispersion relation $\omega_{1}(\mathbf{k})$ of the $1 \mathrm{D} J_{1}-J_{2}$ chain for $J_{1} / J_{2}=0.5$ and $J_{2}>0 . J_{3}=0$ or $k^{a}=k^{b}=0$ is assumed.

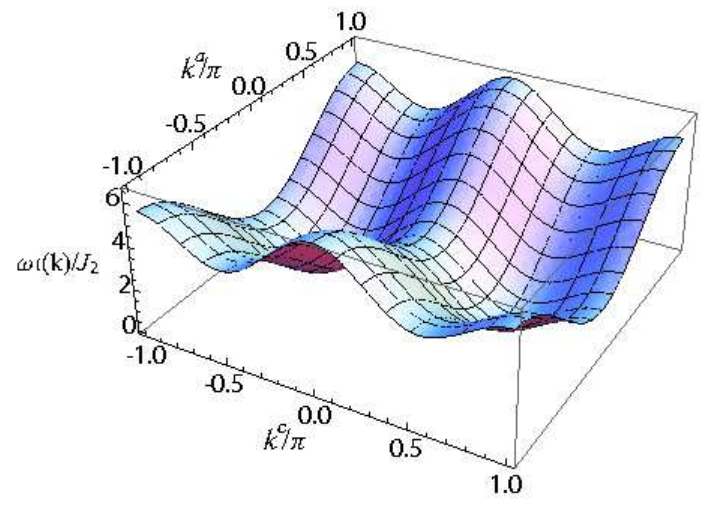

Fig. 5. (Color online) The dispersion relation $\omega_{1}(\mathbf{k})$ of the $1 \mathrm{D} J_{1}-J_{2}$ chains on the cubic lattice for $J_{1} / J_{2}=0.5, J_{2}>0$ and $J_{3} / J_{2}=-2 . k^{c}=0$ is assumed.

\section{$2.3 J_{1}-J_{2}$ model on the square lattice}

The fully saturated phase in the $J_{1}-J_{2}$ model on the square lattice (see Fig. 6) also has the nontrivial dispersion relation:

$$
\epsilon_{2}(\mathbf{k})=J_{1}\left(\cos k^{a}+\cos k^{b}\right)+J_{2}\left(\cos \left(k^{a}+k^{b}\right)+\cos \left(k^{a}-k^{b}\right)\right) .
$$

For $-2<J_{1} / J_{2}<2$ and $J_{2}>0$, the dispersion relation $\omega_{2}(\mathbf{k})$ has two minima at $\mathbf{Q}_{1}^{(2)}=(0, \pi)$ and $\mathbf{Q}_{2}^{(2)}=(\pi, 0)$ as shown in Fig. 7.

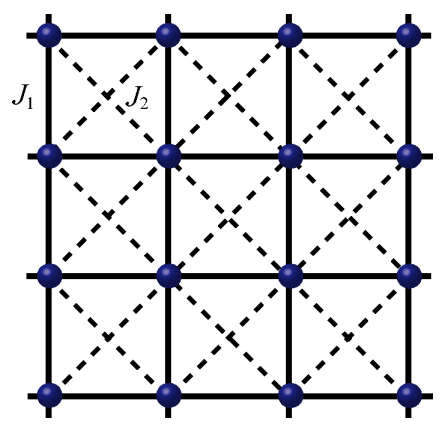

Fig. 6. (Color online) Two dimensional square lattice. The dots represent spins connected by the nearest neighbor Heisenberg exchange coupling $J_{1}$ and the next nearest neighbor coupling $J_{2}$.

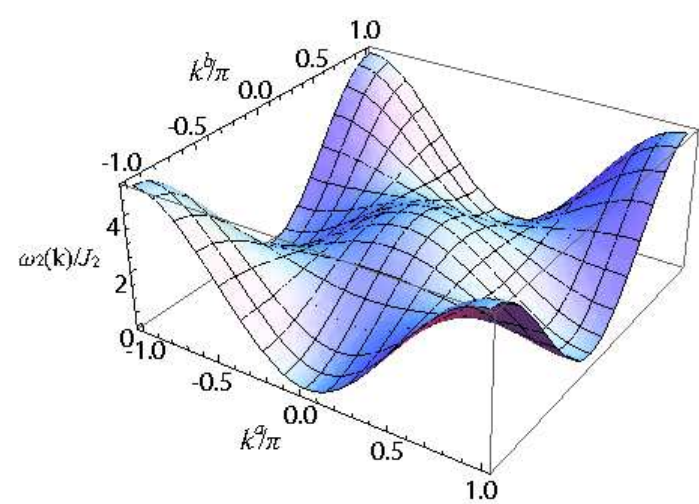

Fig. 7. (Color online) The dispersion relation $\omega_{2}(\mathbf{k})$ for $J_{1} / J_{2}=-0.5$ and $J_{2}>0$ in the $J_{1}-J_{2}$ model on the two-dimensional square lattice.

\section{The Snell's Law in the Ferromagnets Junction System}

In this section, we study the relation of the angles of incident-, reflected- and transmission- spinwaves passing through a boundary (the Snell's law). Let us consider a ferromagnet/ferromagnet junction system whose boundary plane is flat and is perpendicular to $c$ direction. On the boundary the proximity effect may induce a spin-exchange coupling which magnetically relates two ferromagnets. This proximity effect leads to transmission of incident spinwave. The schematic figure of the reflection and refraction process in the usual ferromagnets junction system is shown in Fig. 8.

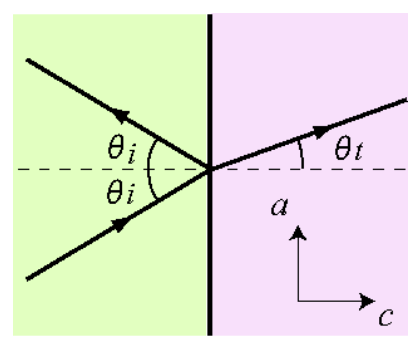

Fig. 8. (Color online) Schematic figure of refraction and reflection process of spinwave in the usual ferromagnets junction system in the case of $k_{b}=0$. The travelling direction of spinwave is determined by the group velocity.

In the case of light, the Snell's law is understood by the conservation law of the energy and that of the momentum parallel to the surface. The same context can be applied to determines the Snell's law of spinwave in the ferromagnets junction system if the following conditions are satisfied: (i) The exchange interactions in each ferromagnet and on the boundary have a rotational symmetry around the $z$ direction in spin space. (ii) Each ferromagnet has a translational symmetry (far away from the boundary). (iii) Both ferromagnets have the same lattice vectors in the $a-b$ plane, and the translational symmetry in the $a-b$ plane exists in the whole junction system. ${ }^{30)}$ The assumption (i) leads to the conservation law of a total number of magnons, and (ii),(iii) lead to the conservation law of a total momentum of the $a$ and $b$ direction. Of course, the momentum conservation law of the $c$ direction does not hold by the boundary effect.

Since the above discussion may be formal, let us consider the simple junction system consists of ferromagnets with the 
nearest neighbor interaction $J\left(J^{\prime}\right)$ on the cubic lattice (see Sec. 2.1) with the same lattice constant. This junction system is schematically illustrated in Fig. 9. It may be appropriate to consider the exchange interaction on the boundary as

$$
\Delta \sum_{a, b} \mathbf{S}_{\mathbf{i}=\left(a, b, c_{1}\right)} \cdot \mathbf{S}_{\mathbf{j}=\left(a, b, c_{2}\right)},
$$

where $\Delta$ is the strength of the exchange interaction on the boundary, and $c_{1}$ and $c_{2}$ is the position of the boundary in the c direction. This boundary condition satisfies the conditions (i), (iii). ${ }^{31)}$

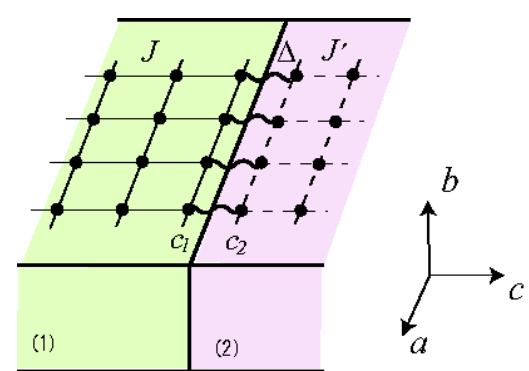

Fig. 9. (Color online) Junction system of usual ferromagnets on the cubic lattice. The dots represent spins. The exchange coupling $\Delta$ due to the quantum proximity effect is considered on the boundary.

Let us consider the case that the incident spinwave in the medium (1) has the momentum $\mathbf{k}_{i}$. The incident spinwave arriving at a boundary is divided into the transmission wave of $\mathbf{k}_{t}$ and the reflected wave of $\mathbf{k}_{r}$.

The assumption (i) leads to the energy conservation

$$
\Omega=\omega_{(1)}\left(\mathbf{k}_{i}\right)-\mu_{(1)}=\omega_{(1)}\left(\mathbf{k}_{r}\right)-\mu_{(1)}=\omega_{(2)}\left(\mathbf{k}_{t}\right)-\mu_{(2)} .
$$

As discussed in Appendix, the group velocity corresponds to the current of magnons. Hence, it is appropriate in our setting to assume that the group velocity of the transmission (reflection) wave has the same (opposite) direction along the $\mathrm{c}$ axis as that of the incident wave. Namely,

$v_{g}^{(1) c}\left(\mathbf{k}_{i}\right) /\left|v_{g}^{(1) c}\left(\mathbf{k}_{i}\right)\right|=-v_{g}^{(1) c}\left(\mathbf{k}_{r}\right) /\left|v_{g}^{(1) c}\left(\mathbf{k}_{r}\right)\right|=v_{g}^{(2) c}\left(\mathbf{k}_{r}\right) /\left|v_{g}^{(2) c}\left(\mathbf{k}_{r}\right)\right|$.

In addition, the momentum conservation law parallel to the boundary leads to

$$
k_{i}^{a}=k_{r}^{a}=k_{t}^{a}, k_{i}^{b}=k_{r}^{b}=k_{t}^{b} .
$$

These equations determine $\mathbf{k}_{r}$ and $\mathbf{k}_{t}$, which lead to the Snell's law. In general, the transmission- and reflected spinwaves determined by eqs. (12), (13), (14) are not necessarily single valued.

Finally, let us discuss the general relation between the reflectance and transmittance if the conditions (i) (ii) (ii) are satisfied. By labeling spinwaves as $\mathbf{k}^{(1)}, \mathbf{k}^{(2)}, \mathbf{k}^{(3)} \cdots$, this reflection and refraction process can be written as the ket

$$
\left(A \alpha_{\mathbf{k}_{i}}^{(1) \dagger}+\sum_{j} B_{j} \alpha_{\mathbf{k}_{r}^{(j)}}^{(1) \dagger}+\sum_{j} C_{j} \alpha_{\mathbf{k}_{t}^{(j)}}^{(2)}\right)|0\rangle,
$$

where $|0\rangle$ is vacuum state and $a^{(1,2)}(\mathbf{k})$ approaches the free boson in the media $(1,2)$ with the momentum $\mathbf{k}$ sufficiently away from the boundary. $\left|v_{g}^{(1) c}\left(\mathbf{k}_{r}^{(j)}\right) / v_{g}^{(1) c}\left(\mathbf{k}_{i}\right) \| B_{j} / A\right|^{2}$ $\left(\left|v_{g}^{(2) c}\left(\mathbf{k}_{t}^{(j)}\right) / v_{g}^{(1) c}\left(\mathbf{k}_{i}\right)\right|\left|C_{j} / A\right|^{2}\right)$ is reflectance (transmittance). By considering the cuboid discussed in Appendix, we obtain the magnon number conservation law:

$$
|A|^{2} v_{g}^{(1) c}\left(\mathbf{k}_{i}\right)+\sum_{j}\left|B_{j}\right|^{2} v_{g}^{(1) c}\left(\mathbf{k}_{r}^{(j)}\right)=\sum_{j}\left|C_{j}\right|^{2} v_{g}^{(2) c}\left(\mathbf{k}_{t}^{(j)}\right) \text {. }
$$

The concrete expression of $B_{j}$ and $C_{j}$ will be discussed in Sec. 4 by explicitly considering the boundary condition.

Since we have discussed the procedure to find the Snell's law, let us study the refraction processes in the various ferromagnets junction systems.

\section{1 usual case}

First, let us further study the Snell's law of the junction system of the usual ferromagnets on the cubic lattice with the nearest neighbor exchange coupling $J<0\left(J^{\prime}<0\right)$ as illustrated in Fig. 9. The dispersion relation on each ferromagnet is respectively given by

$$
\begin{aligned}
& \omega_{s 1}(\mathbf{k})=2 S\left(J\left(\cos k^{a}+\cos k^{b}+\cos k^{c}\right)-3 J\right), \\
& \omega_{s 2}(\mathbf{k})=2 S\left(J^{\prime}\left(\cos k^{a}+\cos k^{b}+\cos k^{c}\right)-3 J^{\prime}\right),
\end{aligned}
$$

where $\mu_{1}<0$ and $\mu_{2}<0 . \mu_{1,2}$ are given in eq. (4). In these usual ferromagnets, the group velocity $\mathbf{v}_{g}$, which carry the angular momentum, is given by

$$
\begin{aligned}
& \mathbf{v}_{g}^{(s 1)}(\mathbf{k})=2 S \nabla_{\mathbf{k}} \epsilon(\mathbf{k})=-2 S J\left(\sin k^{a}, \sin k^{b}, \sin k^{c}\right), \\
& \mathbf{v}_{g}^{(s 2)}(\mathbf{k})=-2 S J^{\prime}\left(\sin k^{a}, \sin k^{b}, \sin k^{c}\right) .
\end{aligned}
$$

The sign of the group velocity $v_{g}^{(s 1) a, b, c}$ is always the same as the sign of $k^{a, b, c}$ for $-\pi<k^{a, b, c}<\pi$. If we consider the incident wave of wavevector $\mathbf{k}_{i}$, eqs. (12), (13), (14) uniquely determine the reflected wave of $\mathbf{k}_{r}$ and the transmission wave of $\mathbf{k}_{t}$. We obtain

$$
\begin{aligned}
\cos k_{t}^{c}= & \frac{1}{J^{\prime}}\left(J \cos k_{i}^{c}+\left(J-J^{\prime}\right)\left(\cos k_{i}^{a}+\cos k_{i}^{b}\right)-3\left(J-J^{\prime}\right)\right. \\
& \left.+\frac{\mu_{2}-\mu_{1}}{2 S}\right),
\end{aligned}
$$

where $k_{t}^{c} /\left|k_{t}^{c}\right|=k_{i}^{c} /\left|k_{i}^{c}\right|$ for $-\pi<k_{t}^{c}<\pi$. This implies that, the larger the chemical potential $\mu_{2}$ is, the slower the group velocity of the transmission wave becomes. Since $\mu_{1,2}$ depends on the external magnetic field $\mathrm{H}_{1,2}$ in each compound, we explicitly see that the propagation of the spinwave can be controlled by the (local) external magnetic field as discussed in Refs. 3-6.

If we consider the long-wavelength limit $|\mathbf{k}| \rightarrow 0$, the Snell's law in this system becomes simple. The group velocity is proportional to the phase velocity in the long wavelength limit $\mathbf{k} \rightarrow 0$. Up to the order of $\mathbf{k}^{2}$, the refractive index is given by

$$
n=\frac{\sin \theta_{i}}{\sin \theta_{t}}=\frac{\left|\mathbf{k}_{t}\right|}{\left|\mathbf{k}_{c}\right|}=\sqrt{\frac{\Omega-J^{\prime}+\mu_{2}}{\Omega-J+\mu_{1}}},
$$

where $\theta_{i}\left(\theta_{t}\right)$ is the angle of incidence (refraction) as shown in Fig. 8, and $\Omega$ is given in eq. (12). Eq. (20) is exactly the same as the result obtained in Ref. 4. 


\section{2 $J_{1}-J_{2}$ chains parallel to the boundary}

Next, let us consider the reflection and refraction process of spinwaves in the usual ferromagnet/frustrated ferromagnet junction system. In this subsection, as a concrete example of frustrated ferromagnets, we consider the $J_{1}-J_{2}$ chains on the cubic lattice discussed in Sec. 2.2. We assume that the $J_{1}-J_{2}$ chains are parallel to the boundary plane as shown in Fig. 10.

The dispersion relation and the group velocity in the $J_{1}-J_{2}$ chains on the cubic lattice are given by

$$
\begin{aligned}
\omega_{11}(\mathbf{k})= & 2 S\left(J_{1} \cos k^{a}+J_{2} \cos 2 k^{a}\right. \\
& \left.+J^{\prime}\left(\cos k^{b}+\cos k^{c}-2\right)-\frac{J_{1}^{2}}{8 J_{2}}-J_{2}\right), \\
\mathbf{v}_{g}^{(11)}(\mathbf{k})= & -2 S\left(J_{1} \sin k^{a}+2 J_{2} \sin 2 k^{a}, J^{\prime} \sin k^{b}, J^{\prime} \sin k^{c}\right) .
\end{aligned}
$$

If we consider the incident wave of wavevector $\mathbf{k}_{i}$ in the usual ferromagnet, the transmission wave of $\mathbf{k}_{t}$ is uniquely given by eqs. (12), (13), (14):

$$
\begin{aligned}
\cos k_{t}^{c} & =\frac{1}{J^{\prime}}\left(\frac{\Omega+\mu_{2}}{2 S}-J_{1} \cos k_{i}^{a}-J_{2} \cos 2 k_{i}^{a}\right. \\
& \left.-J^{\prime}\left(\cos k_{i}^{b}-1\right)-\frac{J_{1}^{2}}{8 J_{2}}-J_{2}\right),
\end{aligned}
$$

where $k_{t}^{c} /\left|k_{t}^{c}\right|=k_{i}^{c} /\left|k_{i}^{c}\right|$ for $-\pi<k_{t}^{c}<\pi$. As discussed in Sec 2.2 , if $-4<J_{1} /\left|J_{2}\right|<4$ and $J_{2}>0$, the equation $J_{1} \cos k_{i}^{a}+J_{2} \cos 2 k_{i}^{a}$ has two minima at $\cos k_{i}^{a}=-\frac{J_{1}}{4 J_{2}}$. If $\cos k_{i}^{a}>-\frac{J_{1}}{4 J_{2}}$, the $a$ component of the group velocities of the incident spinwave is of the opposite sign to that of the transmission spinwave: the refractive index becomes negative ${ }^{32)}$ as illustrated in Fig. 11.

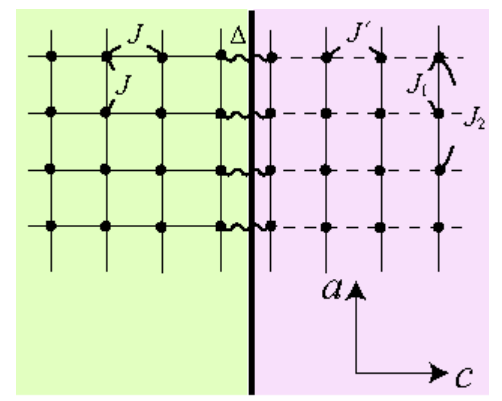

Fig. 10. (Color online) Junction system of the usual ferromagnet (left, green) /ferromagnet consisting of $J_{1}-J_{2}$ chains which lie along the $a$ axis (right, red). The exchange coupling $\Delta$ due to the quantum proximity effect is considered on the boundary.

\section{$3.3 J_{1}-J_{2}$ chains perpendicular to the boundary}

In this subsection, we study the case that the frustrated ferromagnet consists of $J_{1}-J_{2}$ chains perpendicular to the boundary plane as shown in Fig. 12. The dispersion relation in the $J_{1}-J_{2}$ chains is given by

$$
\begin{aligned}
\omega_{12}(\mathbf{k}) & =2 S\left(J_{1} \cos k^{c}+J_{2} \cos 2 k^{c}\right. \\
& \left.+J^{\prime}\left(\cos k^{a}+\cos k^{b}-2\right)+\frac{J_{1}^{2}}{8 J_{2}}+J_{2}\right) .
\end{aligned}
$$

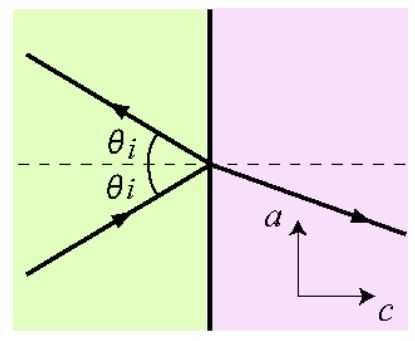

Fig. 11. (Color online) Schematic figure of refraction and reflection process of spinwave in the case of the negative refraction index and $\mathbf{k}_{c}=0$. The travelling direction of spinwave is determined by the group velocity.

If the spinwave of $\mathbf{k}_{i}$ in the usual ferromagnet is injected into the $J_{1}-J_{2}$ chains, the transmission waves of $\mathbf{k}_{t}^{( \pm)}$are given by eqs. (12), (13), (14):

$$
\cos k_{t}^{( \pm) c}= \pm \sqrt{\frac{\Omega-2 S J^{\prime}\left(\cos k^{a}+\cos k^{b}-2\right)+\mu_{2}}{4 S J_{2}}}-\frac{J_{1}}{4 J_{2}},
$$

where $-\frac{J_{1} \sin k_{t}^{( \pm c}+2 J_{2} \sin 2 k_{t}^{( \pm c c}}{\left|J_{1} \sin k_{t}^{( \pm c}+2 J_{2} \sin 2 k_{t}^{ \pm c}\right|}=\frac{k_{i}^{c}}{\left|k_{i}^{c}\right|}$. The appearance of \pm in the right-hand side of (24) is because the dispersion relation has two minima in the direction of $\mathrm{c}$ axis. Hence, when both $\mathbf{k}^{ \pm}$ are permitted, two species of transmission spinwave appear as shown in Fig. 13.

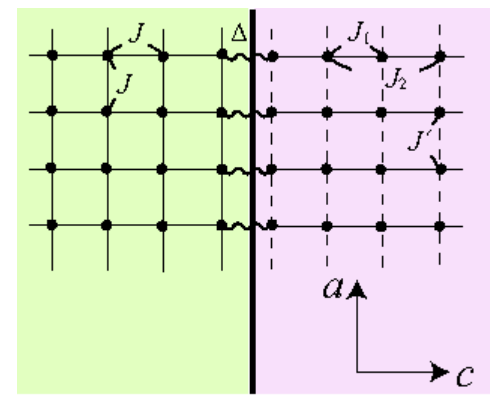

Fig. 12. (Color online) Junction system of the usual ferromagnet (left, green) /ferromagnet consisting of the $J_{1}-J_{2}$ chains which lie along the $c$ axis (right, red). The exchange coupling $\Delta$ due to the quantum proximity effect is considered on the boundary.

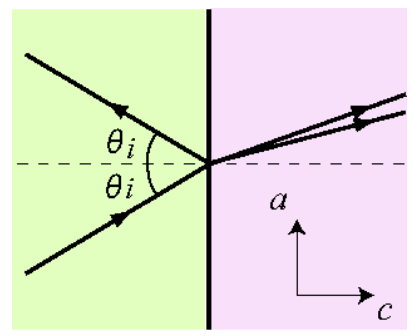

Fig. 13. (Color online) Schematic figure of refraction and reflection process of spinwave in the case of $\mathbf{k}_{b}=0$. The incident spinwave can split into two transmission waves. The travelling direction of spinwave is determined by the group velocity. 


\section{$3.4 \quad J_{1}-J_{2}$ model on the square lattice}

Finally, let us briefly consider the fully polarized phase in the $J_{1}-J_{2}$ model on the square lattice as an example of frustrated ferromagnets. We study the 2-dimensional junction system in the $a-b$ plane shown in Fig. 14. The dispersion relation in the $J_{1}-J_{2}$ model is given by eq. (10). In the case that $-2<J_{1} / J_{2}<2$ and $J_{2}>0$, by injecting the spinwave of $\mathbf{k}_{i}$ from the usual ferromagnet, the transmission spinwave of $\mathbf{k}_{t}$ is given by

$$
\begin{aligned}
k_{t}^{a} & =k_{i}^{a}, \\
\cos k_{t}^{b} & =\frac{1}{J_{1}+2 J_{2} \cos k_{i}^{a}}\left(\frac{\Omega+\mu_{2}}{2 S}-J_{1} \cos k_{i}^{a}-2 J_{2}\right),
\end{aligned}
$$

where $-\frac{J_{1} \sin k_{t}^{b}+2 J_{2} \cos k_{t}^{a} \sin k_{t}^{b}}{\left|J_{1} \sin k_{t}^{b}+2 J_{2} \cos k_{t}^{a} \sin k_{t}^{b}\right|}=-\frac{\sin k_{i}^{b}}{\left|\sin k_{i}^{b}\right|}$. The negative refraction can be realized as well as the junction system discussed in Sec. 3.2 as illustrated in Fig. 11.

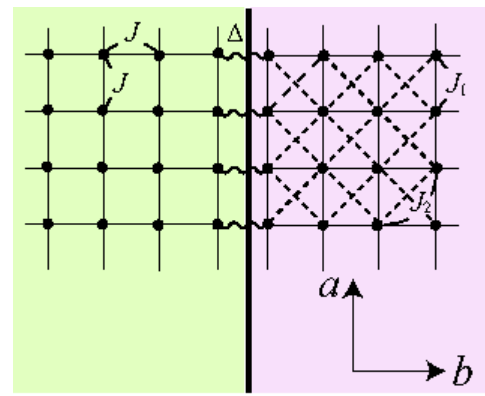

Fig. 14. (Color online) Junction system of the usual ferromagnet (left, green) /ferromagnet consisting of the $J_{1}-J_{2}$ model on the square lattice (right, red). The exchange coupling $\Delta$ due to the quantum proximity effect is considered on the boundary.

\section{Reflectance and Transmittance}

We have studied the relation between the refraction angles of transmission spinwaves and the angle of incident spinwave, namely, the Snell's law of spinwaves in ferromagnets junction system. In this section, let us discuss the reflectance and the transmittance of spinwaves. Although in this section we focus on the concrete models discussed in Secs. 3.1, 3.2, 3.3, our approach will be easily applied to other frustrated ferromagnets junction systems satisfying the conditions (i), (ii), (iii) discussed in Sec. 3.

\section{1 usual case}

Let us study the reflectance and the transmittance of spinwaves in the usual ferromagnet/ferromagnet junction system on the cubic lattice with the boundary condition (11) (see Fig. 9). The Hamiltonian of the total junction system is given by

$$
\begin{aligned}
& H_{\text {tot1 }}=\sum_{\langle\mathbf{i}, \mathbf{j}\rangle \text { for } i_{c}, j_{c} \leq 0} J \mathbf{S}_{\mathbf{i}} \cdot \mathbf{S}_{\mathbf{j}}+\sum_{i_{c} \leq 0}\left(-K_{1}\left(S_{\mathbf{i}}^{z}\right)^{2}+\mathrm{H}_{1} S_{\mathbf{i}}^{z}\right) \\
& +\sum_{\langle\mathbf{i}, \mathbf{j}\rangle \text { for } i_{c}, j_{c} \geq 1} J^{\prime} \mathbf{S}_{\mathbf{i}} \cdot \mathbf{S}_{\mathbf{j}}+\sum_{i_{c} \geq 1}\left(-K_{2}\left(S_{\mathbf{i}}^{z}\right)^{2}+\mathrm{H}_{2} S_{\mathbf{i}}^{z}\right) \\
& +\Delta \sum_{a, b} \mathbf{S}_{\mathbf{i}=(a, b, c=0)} \cdot \mathbf{S}_{\mathbf{j}=(a, b, c=1)}
\end{aligned}
$$

where we assume that the boundary is located between $c=0$ and 1 , and $\langle i, j\rangle$ represents the pairs of the nearest neighbor coupling. The reflection and refraction process with the incident spinwave of $\mathbf{k}_{i}$ may be written as the ket

$$
\begin{aligned}
\left|R R_{1}\right\rangle= & \sum_{a, b} e^{i\left(k_{i}^{a} a+k_{i}^{b} b\right)}\left(A \sum_{c=-\infty}^{0} e^{i k_{i}^{c} c} \alpha_{a, b, c}^{\dagger}+B \sum_{c=-\infty}^{0} e^{-i k_{r}^{c} c} \alpha_{a, b, c}^{\dagger}\right. \\
& \left.+C \sum_{c=1}^{\infty} e^{i k_{t}^{c}(c-1)} \alpha_{a, b, c}^{\dagger}\right)|0\rangle,
\end{aligned}
$$

where $k_{t}^{c}$ is given by eq. (19). Then, $A, B, C$ are determined by the Schröedinger equation:

$$
H_{\text {tot } 1}\left|R R_{1}\right\rangle=\Omega\left|R R_{1}\right\rangle .
$$

If we consider the transition matrix element of $H_{\text {tot } 1}\left|R R_{1}\right\rangle$ to a lattice position, (28) is always satisfied except at $c=0,1$. Hence, we consider

$$
\begin{aligned}
& \left\langle 0\left|a_{a, b, c=0} H_{\mathrm{tot} 1}\right| R R_{1}\right\rangle=\Omega(A+B) e^{i\left(k_{i}^{a} a+k_{i}^{b} b\right)}, \\
& \left\langle 0\left|a_{a, b, c=1} H_{\mathrm{tot} 1}\right| R R_{1}\right\rangle=\Omega C e^{i\left(k_{i}^{a} a+k_{i}^{b} b\right)} .
\end{aligned}
$$

Equivalently,

$$
\begin{aligned}
& \left(\xi_{1}\left(k_{i}^{c}\right)-J e^{-i k_{i}^{c}}\right) A+\left(\xi_{1}\left(k_{i}^{c}\right)-J e^{i k_{i}^{c}}\right) B=\Delta C, \\
& \Delta(A+B)=\left(\xi_{2}\left(k_{t}^{c}\right)-J^{\prime} e^{i k_{t}^{c}}\right) C,
\end{aligned}
$$

where

$$
\begin{aligned}
& \xi_{1}\left(k_{i}^{c}\right)=2 J\left(\cos k_{i}^{c}-1\right)-J+\Delta, \\
& \xi_{2}\left(k_{t}^{c}\right)=2 J^{\prime}\left(\cos k_{t}^{c}-1\right)-J^{\prime}+\Delta .
\end{aligned}
$$

Hence, we obtain

$$
\begin{aligned}
& \frac{B}{A}=-\frac{\left(\xi_{1}\left(k_{i}^{c}\right)-J e^{-i k_{i}^{c}}\right)\left(\xi_{2}\left(k_{t}^{c}\right)-J^{\prime} e^{i k_{t}^{c}}\right)-\Delta^{2}}{\left(\xi_{1}\left(k_{i}^{c}\right)-J e^{i k_{i}^{c}}\right)\left(\xi_{2}\left(k_{t}^{c}\right)-J^{\prime} e^{i k_{t}^{c}}\right)-\Delta^{2}}, \\
& \frac{C}{A}=-\Delta \frac{2 i J \sin k_{i}^{c}}{\left(\xi_{1}\left(k_{i}^{c}\right)-J e^{i k_{i}^{c}}\right)\left(\xi_{2}\left(k_{t}^{c}\right)-J^{\prime} e^{i k_{t}^{c}}\right)-\Delta^{2}} .
\end{aligned}
$$

By using the relation

$$
\begin{aligned}
& \left|\left(\xi_{1}\left(k_{i}^{c}\right)-J e^{-i k_{i}^{c}}\right)\left(\xi_{2}\left(k_{t}^{c}\right)-J^{\prime} e^{i k_{t}^{c}}\right)-\Delta^{2}\right|^{2} \\
& -\left|\left(\xi_{1}\left(k_{i}^{c}\right)-J e^{i k_{i}^{c}}\right)\left(\xi_{2}\left(k_{t}^{c}\right)-J^{\prime} e^{i k_{t}^{c}}\right)-\Delta^{2}\right|^{2} \\
& =4 J J^{\prime} \Delta^{2} \sin k_{i}^{c} \sin k_{t}^{c}=\Delta^{2} v_{g}^{(s 1)}\left(\mathbf{k}_{i}\right) v_{g}^{(s 2)}\left(\mathbf{k}_{t}\right) / S^{2},
\end{aligned}
$$

the magnon-number-conservation law given by eq. (16) is easily confirmed as

$$
v_{g}^{(s 1) c}\left(\mathbf{k}_{i}\right)\left(1-\left|\frac{B}{A}\right|^{2}\right)=v_{g}^{(s 2) c}\left(\mathbf{k}_{t}\right)\left|\frac{C}{A}\right|^{2},
$$

regardless of any $\mathbf{k}_{i}$ and $\mathbf{k}_{t}$. From eq. (32), we explicitly find the dependence of the transmittance and the phase shift of spinwave on the boundary condition $\Delta$. For example, the transmittance $\left|v_{g}^{(s 2) c}\left(\mathbf{k}_{t}\right) / v_{g}^{(s 1) c}\left(\mathbf{k}_{i}\right)\right||C / A|^{2}$, the reflectance $|B / A|^{2}$ and the refraction index $\sin \theta_{i} / \sin \theta_{t}$ in the specific case are shown in Fig. 15. Since the travelling direction of spinwave is given by the group velocity, incident spinwave of different frequencies could have the same incident angle. For $\Delta \rightarrow 0$, the transmittance leads: $|C / A|^{2}=O\left(\Delta^{2} / J^{2}\right)$. 


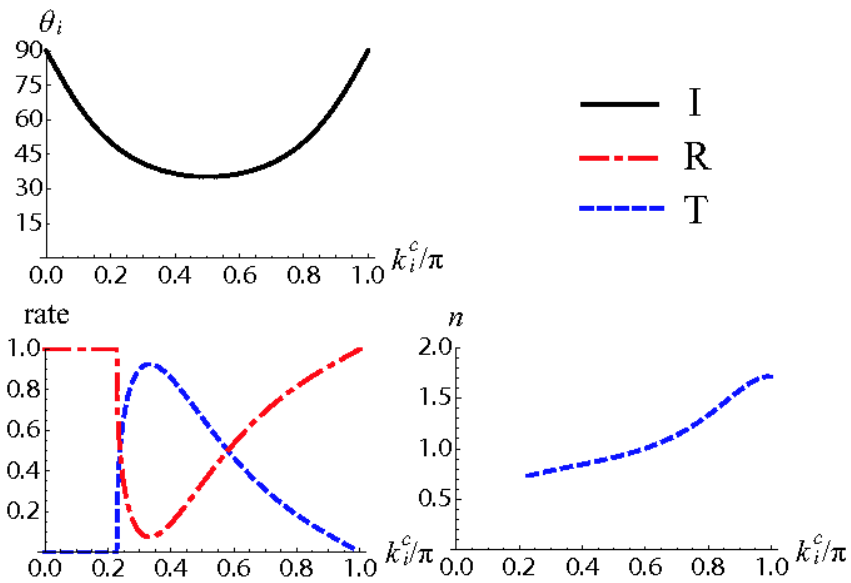

Fig. 15. (Color online) The incident angle $\theta_{i}$, the transmittance, the reflectance (rate), and the refraction index $n=\sin \theta_{i} / \sin \theta_{t}$ in the usual ferromagnet/ferromagnet junction system for $J=-1, J^{\prime}=-1.5, \Delta=-1.5$, $\mu_{1} / 2 S=-1.6, \mu_{2} / 2 S=-1.7$. The incident spinwave with $k_{a}=\pi / 4$ and $k_{b}=0$ in the usual ferromagnet is assumed. $I, R, T$ respectively denotes the incident wave, the reflection wave and the transmission wave. The incident angle and the refraction index are determined by the direction of the group velocity.

\section{$4.2 J_{1}-J_{2}$ chains parallel to the boundary}

Next, let us study the case that one side of the ferromagnetsjunction system consists of the $J_{1}-J_{2}$ chains parallel to the boundary as shown in Fig. 10. The reflection and refraction process is described by the following ket:

$$
\begin{aligned}
\left|R R_{2}\right\rangle & =\sum_{a, b} e^{i\left(k_{i}^{a} a+k_{i}^{b} b\right)}\left(A \sum_{c=-\infty}^{0} e^{i k k_{i}^{c} c} \alpha_{a, b, c}^{\dagger}+B \sum_{c=-\infty}^{0} e^{-i k_{r}^{c} c} \alpha_{a, b, c}^{\dagger}\right. \\
& \left.+C \sum_{c=1}^{\infty} e^{i k_{t}^{c}(c-1)} \alpha_{a, b, c}^{\dagger}\right)|0\rangle
\end{aligned}
$$

where $k_{t}^{c}$ is given by eq. (22). The procedure to determine $B, C$ is exactly the same as that of the previous section. Hence, $B$ and $C$ are given by (32). For exapmle, the transmittance $\left|v_{g}^{(11) c}\left(\mathbf{k}_{t}\right) / v_{g}^{(s 1) c}\left(\mathbf{k}_{i}\right)\right||C / A|^{2}$, the reflectance $|B / A|^{2}$ and the refraction index in the specific case are shown in Fig. 16, where the occurrence of the negative refraction index is explicitly seen.

\section{$4.3 J_{1}-J_{2}$ chains perpendicular to the boundary}

Finally, we consider the junction system of the $J_{1}-J_{2}$ chains perpendicular to the boundary as shown in Fig. 12. In this case, the ket is given by

$$
\begin{aligned}
& \left|R R_{3}\right\rangle=\sum_{a, b} e^{i\left(k_{i}^{a} a+k_{i}^{b} b\right)}\left(A \sum_{c=-\infty}^{0} e^{i k_{i}^{c} c} \alpha_{a, b, c}^{\dagger}+B \sum_{c=-\infty}^{0} e^{-i k_{r}^{c} c} \alpha_{a, b, c}^{\dagger}\right. \\
& \left.+C_{1} \sum_{c=1}^{\infty} e^{i k_{t}^{(+) c}(c-1)} \alpha_{a, b, c}^{\dagger}+C_{2} \sum_{c=1}^{\infty} e^{i k_{t}^{(-) c}(c-1)} \alpha_{a, b, c}^{\dagger}\right)|0\rangle
\end{aligned}
$$

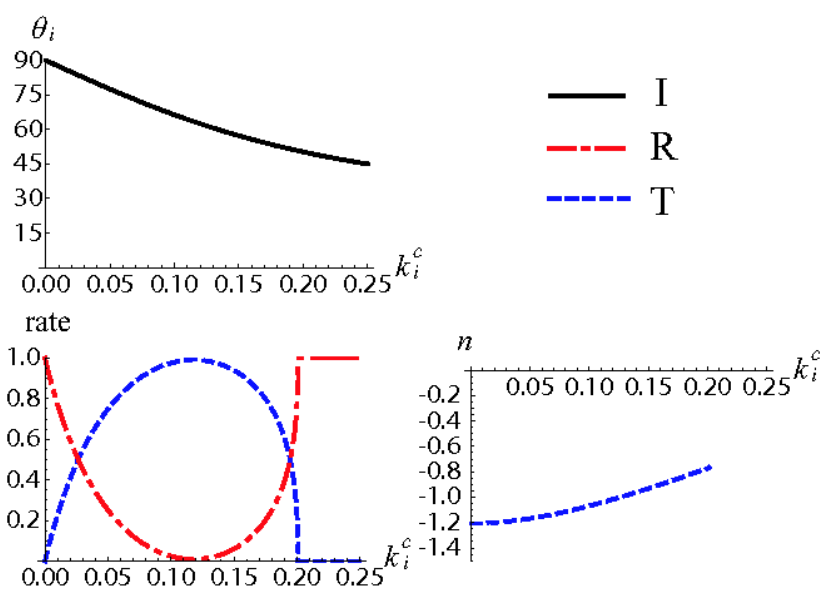

Fig. 16. (Color online) The incident angle $\theta_{i}$, the transmittance, the reflectance (rate), and the refraction index $n$ in the usual ferromagnet/ frustrated ferromagnet junction system consisting of the $J_{1}-J_{2}$ chains parallel to the boundary plane for $J=-1, J_{1}=-2, J_{2}=1, J_{3}=-0.5, \Delta=-1.0$, $\mu_{1} / 2 S=-1.6, \mu_{2} / 2 S=-1.5$. The incident spinwave with $k_{a}=\pi / 4$ and $k_{b}=0$ in the usual ferromagnet is assumed. $I, R, T$ respectively denotes the incident wave, the reflection wave and the transmission wave. The incident angle and the refraction index are determined by the direction of the group velocity.

where $k_{t}^{( \pm) c}$ are given by eq. (24). We see that (28) is satisfied at any lattice cite except at $c=0,1,2$. Hence, we consider

$$
\begin{aligned}
& \left\langle 0\left|a_{a, b, c=0} H_{\mathrm{tot} 3}\right| R R_{1}\right\rangle=\Omega(A+B) e^{i\left(k_{i}^{a} a+k_{i}^{b} b\right)}, \\
& \left\langle 0\left|a_{a, b, c=1} H_{\mathrm{tot} 3}\right| R R_{1}\right\rangle=\Omega\left(C_{1}+C_{2}\right) e^{i\left(k_{i}^{a} a+k_{i}^{b} b\right)}, \\
& \left\langle 0\left|a_{a, b, c=2} H_{\mathrm{tot} 3}\right| R R_{1}\right\rangle=\Omega\left(C_{1} e^{i k_{t}^{(+) c}}+C_{2} e^{i k_{t}^{(-) c}}\right) e^{i\left(k_{i}^{a} a+k_{i}^{b} b\right)},
\end{aligned}
$$

where $H_{\text {tot3 }}$ is the Hamiltonian of the total system considered in this subsection. Equivalently,

$$
\begin{aligned}
& \left(\xi_{1}^{\prime}\left(k_{i}^{c}\right)-J e^{-i k_{i}^{c}}\right) A+\left(\xi_{1}^{\prime}\left(k_{i}^{c}\right)-J e^{i k_{i}^{c}}\right) B=\Delta\left(C_{1}+C_{2}\right), \\
& \left(\xi_{2}^{\prime}\left(k_{t}^{(+) c}\right)-J_{1} e^{i k_{t}^{(+) c}}-J_{2} e^{i 2 k_{t}^{(+) c}}\right) C_{1} \\
& +\left(\xi_{2}^{\prime}\left(k_{t}^{(+) c}\right)-J_{1} e^{i k_{t}^{(-) c}}-J_{2} e^{i 2 k_{t}^{(-) c}}\right) C_{2}=\Delta(A+B), \\
& \left(\xi_{3}^{\prime}\left(k_{t}^{(+) c}\right)-2 J_{1} \cos k_{t}^{(+) c}-J_{2} e^{i 2 k_{t}^{(+) c}}\right) C_{1} e^{i k_{t}^{(+) c}} \\
& +\left(\xi_{3}^{\prime}\left(k_{t}^{(+) c}\right)-2 J_{1} \cos k_{t}^{(-) c}-J_{2} e^{i 2 k_{t}^{(-) c}}\right) C_{2} e^{i k_{t}^{(-) c}}=0,
\end{aligned}
$$

where

$$
\begin{aligned}
\xi_{1}^{\prime}\left(k_{i}^{c}\right)=2 J\left(\cos k_{i}^{c}-1\right)-J+\Delta, \\
\xi_{2}^{\prime}\left(k_{t}^{(+) c}\right)=\xi_{2}^{\prime}\left(k_{t}^{(-) c}\right)=2\left(J_{1} \cos k_{t}^{(+) c}+J_{2} \cos 2 k_{t}^{(+) c}+\frac{J_{1}^{2}}{8 J_{2}}\right) \\
\quad-J_{1}+J_{2}+\Delta, \\
\xi_{3}^{\prime}\left(k_{t}^{(+) c}\right)=\xi_{2}^{\prime}\left(k_{t}^{(+) c}\right)+J_{1}-\Delta .
\end{aligned}
$$

$B / A, C_{1} / A, C_{2} / A$ are given by solving these equations. We numerically confirmed the following magnon number conservation law in the various concrete parameters:

$$
v_{g}^{(s 1) c}\left(\mathbf{k}_{i}\right)\left(1-\left|\frac{B}{A}\right|^{2}\right)=v_{g}^{(12) c}\left(\mathbf{k}_{t}^{(+)}\right)\left|\frac{C_{1}}{A}\right|^{2}+v_{g}^{(12) c}\left(\mathbf{k}_{t}^{(-)}\right)\left|\frac{C_{2}}{A}\right|^{2},
$$


where $v_{g}^{(12) c}(\mathbf{k})=-2 S\left(J_{1} \sin k^{c}+J_{2} \sin 2 k^{c}\right)$. For example, the transmittance $\left|v_{g}^{(12) c}\left(\mathbf{k}_{t}^{+}\right) / v_{g}^{(s 1) c}\left(\mathbf{k}_{i}\right) \| C_{1} / A\right|^{2}$, $\left|v_{g}^{(12) c}\left(\mathbf{k}_{t}^{-}\right) / v_{g}^{(s 1) c}\left(\mathbf{k}_{i}\right)\right|\left|C_{2} / A\right|^{2}$, the reflectance $|B / A|^{2}$ and the refraction index in the specific case are shown in Fig. 17, where two species of transmission wave appear when both $k_{t}^{( \pm) c} \mathrm{~s}$ in eq. (24) have real values.

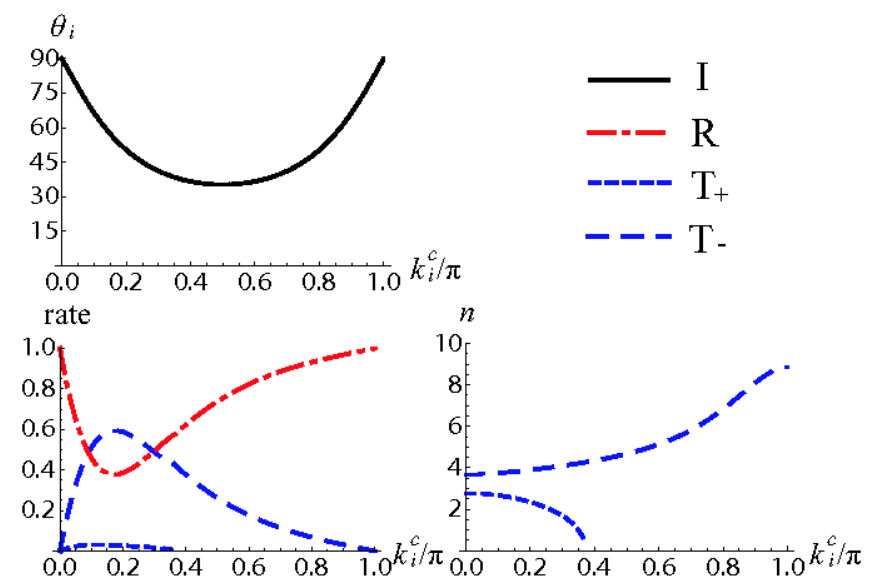

Fig. 17. (Color online) The incident angle $\theta_{i}$, the transmittance, the reflectance (rate), and the refraction index $n$ in the usual ferromagnet/ frustrated ferromagnet junction system consisting of the $J_{1}-J_{2}$ chains perpendicular to the boundary plane for $J=-1, J_{1}=-1.5, J_{2}=1, J_{3}=-0.5, \Delta=-1.0$, $\mu_{1} / 2 S=-1.6, \mu_{2} / 2 S=-1.5$. The incident spinwave with $k_{a}=\pi / 4$ and $k_{b}=0$ in the usual ferromagnet is assumed. $I, R, T_{ \pm}$respectively denotes the incident wave, the reflection wave and the transmission wave with the wavevector $k_{t}^{( \pm) c}$ in eq. (24). The incident angle and the refraction index are determined by the direction of the group velocity.

\section{Conclusion}

We studied the reflection and refraction process of spinwaves in the ferromagnet/frustrated ferromagnet junction system whose ferromagnets are described by the spin Hamiltonian with the generic Heisenberg-exchange coupling, the uniaxial anisotropic interaction and the external magnetic field. By using the Holstein-Primakoff spinwave expansion and taking the large $S$ limit, the ferromagnetic phase is given by the free bosonic Hamiltonian, which describes the dynamics of magnons. If frustration exists, the dispersion relation of magnons (spinwave) can become nontrivial.

In Sec. 3, we discussed the Snell's law of the spinwave in the case that the following conditions are satisfied in the junction system: (i) The exchange interactions in each ferromagnet and on the boundary have a rotational symmetry around the $z$ direction in spin space. (ii) Each ferromagnet has a translational symmetry (far away from the boundary). (iii) Both ferromagnets have the same lattice vectors in the $a-b$ plane, and the translational symmetry in the $a-b$ plane exists in the whole junction system. ${ }^{30)}$ By studying the various junction systems satisfying these conditions, we found the nontrivial refraction process, e.g., the splitting of the spinwave and the negative refraction.

In Sec. 4, we studied the 'reflectance', 'transmittance' and 'phase shift at the boundary' in the concrete examples of the junction systems. Within the large $S$ limit, we exactly obtain these quantities, which explicitly depend on the boundary condition. Throughout this paper our results does not need the long wavelength approximation.

We thank G. Tatara, S. Murakami and A. Yamaguchi for useful discussions.

\section{Appendix: Current of angular momentum}

In this appendix, we briefly review the correspondence between the group velocity of spinwave and the current of angular momentum. We study the spin system described by the Hamiltonian (1). Now, the Hamiltonian (1) commutes with the total spin $S_{\text {tot }}^{z}=\sum_{l} S_{\mathbf{j}}^{z}$. Hence, $S_{\text {tot }}^{z}$ is the conserved quantity. This implies that the total magnon number $\sum_{\mathbf{l}} \alpha_{1}^{\dagger} \alpha_{1}$ conserves, and the conserved current of magnons (related to the U(1) symmetry) exists.

The magnon current is understood by studying in- and outflow of the magnon number in a given region. Let us consider the cuboid $C_{1}$ of which two face-to-face surfaces $S_{1}$ and $S_{2}$ are infinitely large as shown in Fig. A.1.

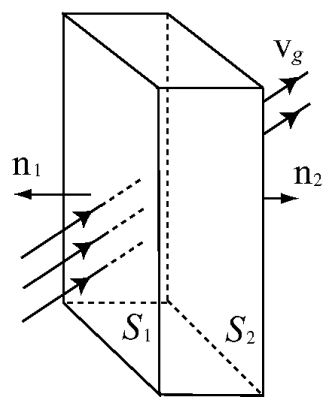

Fig. A.1. Magnon current of the group velocity $v_{g}$ passes through the cuboid $C_{1}$.

The spatially-averaged flow of magnons is determined by the current passing through $S_{1}$ and $S_{2}$. By using the relation

$$
\left[\alpha_{\mathbf{i}}^{\dagger} \alpha_{\mathbf{i}}+\alpha_{\mathbf{j}}^{\dagger} \alpha_{\mathbf{j}}, \mathbf{S}_{\mathbf{i}} \cdot \mathbf{S}_{\mathbf{j}}\right]=0 \text {, }
$$

we obtain

$$
i \frac{d\left(\sum_{l \in C_{1}} \alpha_{\mathbf{1}}^{\dagger} \alpha_{\mathbf{l}}\right)}{d t}=\left[\sum_{l \in C_{1}} \alpha_{\mathbf{1}}^{\dagger} \alpha_{\mathbf{l}}, H\right]=S \sum_{i \in C_{1}, j \notin C_{1}} J_{\mathbf{i j}}\left(\alpha_{\mathbf{i}}^{\dagger} \alpha_{\mathbf{j}}-\alpha_{\mathbf{i}} \alpha_{\mathbf{j}}^{\dagger}\right),
$$

where we use eq. (2). We assume that $J_{\mathbf{i j}}=J_{1}$ is short range so that $J_{\mathbf{l}}=0$ for a large $|\mathbf{I}|$, and that $S_{1}$ is sufficiently separated from $S_{2}$. Then, if we consider the state $|\mathbf{k}\rangle=\alpha_{\mathbf{k}}^{\dagger}|0\rangle$, we obtain

$$
\begin{aligned}
\left\langle\mathbf{k}\left|\frac{d\left(\sum_{l \in C_{1}} \alpha_{\mathbf{1}}^{\dagger} \alpha_{\mathbf{l}}\right)}{d t}\right| \mathbf{k}\right\rangle & =\frac{2 S}{N} \sum_{\mathbf{i} \in C_{1}, \mathbf{j} \in C_{1}} J_{\mathbf{i j}} \sin \mathbf{k} \cdot(\mathbf{i}-\mathbf{j}) \\
& =\frac{1}{N}\left(A_{S_{1}} \mathbf{n}_{1} \cdot \mathbf{v}_{g}(\mathbf{k})+A_{S_{2}} \mathbf{n}_{2} \cdot \mathbf{v}_{g}(\mathbf{k})\right),
\end{aligned}
$$

where $A_{S_{i}}$ is the area of $S_{i}$ and

$$
\mathbf{v}_{g}(\mathbf{k}) \cdot \mathbf{n}=2 S \frac{\partial \epsilon(\mathbf{k})}{\partial \mathbf{k}} \cdot \mathbf{n}=-2 S \sum_{\mathbf{l}} J_{\mathbf{l}} \sin (\mathbf{k} \cdot \mathbf{l}) \mathbf{l} \cdot \mathbf{n} .
$$

These equations imply that the group velocity can be viewed as the magnon current which carry the angular momentum. Even if there is a superposition of various spinwaves 
as $\sum_{l} A_{l} \alpha_{\mathbf{k}_{l}}^{\dagger}|0\rangle$, the time-averaged magnon current is simply summed as

$$
\overline{\mathbf{v}}_{g}^{\mathrm{tot}}=\sum_{l}\left|A_{l}\right|^{2} \mathbf{v}_{g}\left(\mathbf{k}_{l}\right)
$$

1) V. V. Kruglyak, S. O. Demokritov and D. Grundler, J. Phys. D: Appl. Phys. 43264001 (2010).

2) We use the terminology 'trivial dispersion relation' when the dispersion relation is isotropic and the phase velocity is parall to the energy current (e.g., the Poynting vector in the case of light)

3) F. Goedsche, Phys. Status. Solidi, 39 K29 (1970).

4) Y. I. Gorobets and S. A. Reshetnyak, Tech. Phys. 43, 188 (1998)

5) S. A. Reshetnyak, Phys. Solid State, 46, 1061, (2004) [Fiz. Tver. Tela, 46, 1031 (2004)].

6) S. A. Reshetnyak and A. S. Berezhinskiy, The Open Cond. Mat. Phys. Jour., 4, 4, (2011).

7) S-K. Kim, S. Choi, K-S. Lee, D-S. Han, D-E. Jung, and Y-S. Choi, App. Phys. Lett. 92, 212501 (2008).

8) V. E. Demidov, J. Jersch, and S. O. Demokritov, Phys. Rev. B 79, 054417 (2009).

9) V. G. Veselago, Sov. Phys. Usp. 10, 509 (1968) [Usp. Fiz. Nauk 92, 517 (1967)].

10) J. B. Pendry and D. R. Smith, Phys. Today 57, No. 6, 37 (2004).

11) H. T. Diep, Frustrated Spin Systems, edited by H. T. Diep (World Scientific, Singapore, 2004).

12) T. Nagamiya, K. Nagata, and Y. Kitano, Prog. Theor. Phys. 27, 1253 (1962); T. Nagamiya, Solid State Physics, (Academic Press, New York, 1967) 20, 305.

13) M. Hase, H. Kuroe, K. Ozawa, O. Suzuki, H. Kitazawa, G. Kido, and T. Sekine, Phys. Rev. B 70, 104426 (2004).

14) A. V. Chubukov, Phys. Rev. B 44, 4693 (1991).

15) T. Hikihara, L. Kecke, T. Momoi, and A. Furusaki, Phys. Rev. B 78, 144404 (2008).

16) T. Hikihara, T. Momoi, A. Furusaki, and H. Kawamura, Phys. Rev. B 81, 224433 (2010)

17) R. O. Kuzian and S.-L. Drechsler, Phys. Rev. B 75, 024401 (2007).

18) H. T. Ueda, and K. Totsuka, Phys. Rev. B 80, 014417 (2009).

19) A. K. Kolezhuk, F. Heidrich-Meisner, S. Greschner, and T. Vek,
Phys. Rev. B 85, 064420 (2012).

20) D. B. Brown, J. A. Donner, J. W. Hall, S. R. Wilson, R. B. Wilson, D. J. Hodgson, and W. E. Hatfield, Inorg. Chem. 18, 2635 (1979).

21) M. Hagiwara, Y. Narumi, K. Kindo, N. Maeshima, K. Okunishi, T. Sakai, and M. Takahashi, Physica B 294-295, 83 (2001).

22) N. Maeshima, M. Hagiwara, Y. Narumi, K. Kindo, T. C. Kobayashi, and K. Okunishi, J. Phys.: Condens. Matter 15, 3607 (2003).

23) B. J. Gibson, R. K. Kremer, A. V. Prokofiev, W. Assmus, G. J. McIntyre, Physica B 350, e253 (2004).

24) M. Enderle, C. Mukherjee, B. Fåk, R. K. Kremer, J. M. Broto, H. Rosner, S. L. Drechsler, J. Richter, J. Malek, A. Prokofiev, W. Assmus, S. Pujol, J.-L. Raggazzoni, H. Rakoto, M. Rheinstädter and H. M. Rønnow, Europhys. Lett. 70, 237 (2005).

25) L. E. Svistov, T. Fujita, H. Yamaguchi, S. Kimura, K. Omura, A. Prokofiev, A. I. Smirnov, Z. Honda and M. Hagiwara, JETP letters, 93, 21 (2011).

26) R. Nath, A. A. Tsirlin, H. Rosner, and C. Geibel, Phys. Rev. B 78, $064422(2008)$

27) A. A. Tsirlin and H. Rosner, Phys. Rev. B 79, 214417 (2009).

28) P. Thalmeier, M. E. Zhitomirsky, B. Schmidt, and N. Shannon, Phys. Rev. B 77, 104441 (2008)

29) S. Nishimoto, S.-L. Drechsler, R. O. Kuzian, J. van den Brink, J. Richter, W. E. A. Lorenz, Y. Skourski, R. Klingeler and B. Büchner, Phys. Rev. Lett. 107, 097201 (2011).

30) If we consider a junction of different compounds, the assumption (iii) is scarcely satisfied. However, if the difference of the lattice vectors in each compound is small, the deviation from the discussion in our paper may be perturbative and small. In addition, the translational invariance exists within the least common multiple lattice vectors in the $a-b$ plane. Hence, even if the difference of the lattice vectors is large, in some cases our simple discussion may predict the reasonable result, e.g., in the case

of the long wavelength limit.

31) As will be discussed, the refraction angles are independent of the concrete form of the boundary condition as long as the assumptions (i), (iii) hold: the Snell's law is independent of $\Delta$. Only the transmission rate and the phase shift at the boundary depend on $\Delta$ as will be discussed in Sec. 4.1.

32) S. Murakami pointed out the possibility of the negative refraction in the private communication 